\title{
Menstrual Cycle Duration
}

National Cancer Institute

\section{Source}

National Cancer Institute. Menstrual Cycle Duration. NCI Thesaurus. Code C119549.

The length of time of the menses cycle, measured from the beginning of one menstrual period to the beginning of the next. 